\title{
Response and acclimatisation of symptomless smokers on changing to a low tar, low nicotine cigarette
}

\author{
G WOODMAN, S P NEWMAN, D PAVIA, S W CLARKE \\ From the Department of Thoracic Medicine, Royal Free Hospital and School of Medicine, London
}

ABSTRACT Ten symptomless smokers were switched from their usual cigarette to a low tar, low nicotine test cigarette for two weeks to investigate their immediate response and subsequent ${ }_{G}^{N}$ acclimatisation to the test cigarette. The tar $(\mathrm{T})$ and nicotine $(\mathrm{N})$ yields of the test cigarettes werec్ $\mathrm{T}=3.8 \mathrm{mg}, \mathrm{N}=0.6 \mathrm{mg}$; the median yields of the usual cigarettes were $\mathrm{T}=16.4 \mathrm{mg}, \mathrm{N}=1.4 \mathrm{mg}$. $\%$ The subjects were monitored over a six week period comprising a control period (usual cigarette), $\supset$ a test period (test cigarette), and a return period (usual cigarette), each lasting two weeks. The $\vec{z}_{\bar{Z}}$ inhaled smoke volume (smoke from the burning tip of the cigarette which is subsequently inhaled) was measured with a non-invasive radiotracer technique. Puffing indices were recorded using an $\vec{\bullet}$ electronic smoking analyser and flowhead cigarette holder. Measurements were made at the begin- $\infty$ ning of the control period, at the beginning and end of the test period, and at the end of the return period. Subjects kept records of their cigarette consumption during each of the three periods. Aparto from a small change in puff duration, cigarettes were smoked in the same way during the controls and return periods. Mean and total puff volumes increased with the low tar, low nicotine cigaretten but did not change from the beginning to the end of the test period. There was no significant change⿻ between the control, test, and return periods for mean inhaled smoke volume, total inhaled smoke volume, or cigarette consumption. It is concluded that when smokers are switched to a low tar, low3 nicotine cigarette the puff volume increases but there is no change in the inhaled smoke volume or? daily consumption.

The main constituents of cigarette smoke are tar, nicotine, and carbon monoxide. Tar comprises hundreds of chemical compounds including irritant substances that stimulate the secretion of mucus and inhibit mucus clearance, and carcinogens. ${ }^{1}$ Nicotine is a powerful drug and of great psychophysiological importance in smoking; craving for nicotine is regarded as being the prime motive for smoking. Owing to the harmful nature of tar, smokers who will not abstain have been advised to smoke a cigarette brand with a lower tar yield. ${ }^{2}$ There is, however, a strong correlation between the tar and nicotine concentrations of British cigarettes, ${ }^{3}$ so the nicotine yield of a cigarette with a lower tar yield is usually also lower. A standard method of manufacturing ciga-

Address for reprint requests: Mr G Woodman, Department of Thoracic Medicine, Royal Free Hospital, London NW3 2QG.

Accepted 13 October 1986 rettes in the low tar category (up to $11 \mathrm{mg}$ of tar per3. cigarette) is to dilute the smoke by incorporating ven-i tilation holes into the corked paper surrounding the $\frac{3}{3}$ filter tip. It is known that smokers increase the vol-o ume they puff when they are switched from their usual cigarette brand to a weaker one in the low tar cate- $\frac{D}{0}$ gory. ${ }^{45}$ Smokers do not, however, inhale all the smoke puffed, ${ }^{67}$ and puff volume is not a good index $N$ of the amount of smoke inhaled. Carbon monoxiden has a high affinity for haemoglobin, and chronically high carboxyhaemoglobin levels are strongly associ- ated with an increased frequency of atherosclerotic disease. $^{8}$

Two important factors in assessing the health risk to smokers from a particular cigarette are the amount of smoke inhaled and the daily consumption of that cigarette. In an attempt to ascertain indirectly the amount of smoke inhaled, some investigators have $\frac{?}{\mathbb{Q}}$ measured the increase in the concentration of plasma 2 nicotine or its absolute value after smoking. ${ }^{910}$ In the present study a completely non-invasive radiotracero 
technique was used to quantify the inhaled smoke volume before and after switching to low tar, low nicotine cigarettes; measurements of puffing indices and daily cigarette consumption were also made. With data on inhaled smoke volume and information on daily consumption it is possible to infer whether there is a reduction in the exposure of the lung to smoke and a consequent reduction in the health risk to smokers from these cigarettes. Measurements of carbon monoxide levels were not made. Smokers in other switching studies have been allowed lengths of time ranging from 24 hours $^{11}$ to six weeks ${ }^{12}$ to acclimatise to the new cigarette brand. In this study a group of habitual smokers were monitored over a six week period; their initial and longer term response was measured to investigate the validity of inferring the eventual smoking manoeuvre from the first new cigarette smoked. A further aim was to see whether they reverted to their previous smoking habits with their usual brand after a period on a weaker brand.

\section{Methods}

Ten habitual asymptomatic smokers were investigated, eight men and two women. The median (range) age was $24(20-42)$ years, years of smoking seven (3-25), and cigarette yield in $\mathrm{mg}$ : tar 16.5(9-18), nicotine $1 \cdot 4(0 \cdot 9-1 \cdot 5)$, and carbon monoxide $16(10-19)$. The test cigarettes were ventilated and had the following yields; tar $3.8 \mathrm{mg}$, nicotine $0.6 \mathrm{mg}$, and carbon monoxide $4.2 \mathrm{mg}$.

Spirometric lung function tests were performed on all subjects; volumes were corrected to body temperature and pressure saturated with water vapour and the greatest of three measurements used. ${ }^{13}$ Results were expressed as the median (range) percentages of predicted values. ${ }^{1415}$ The forced vital capacity was $108(89-120) \%$ and FEV $_{1}$ $103(86-129) \%$; these were determined using a dry bellows spirometer (Vitalograph). The peak expiratory flow was $90(75-114) \%$, measured with a
Wright peak flow meter. Maximum expiratory flow rates at $50 \%\left(\dot{V}_{m a x}\right)$ and $25 \%\left(\dot{V} \max _{25}\right)$ of vital capacity were $87(57-134)$ and $89(52-154 \%) \%$, respectively, measured using an Ohio 840 spirometer equipped with a Bryans X-Y plotter.

\section{PROTOCOL}

The subjects made a preliminary visit to the laboratory, during which they were familiarised with the procedure, performed lung function tests, and gave details about smoking and health. Subjects were instructed to abstain from smoking for at least one hour before four subsequent visits, which were made at two week intervals. The organisation of the study is shown at the top of table 1 . Subjects were monitored at the beginning (visit C) of a two week control period during which they smoked their usual brand, at the beginning (visit T1) and end (visit T2) of a two week test period during which they smoked the low tar, low nicotine brand, and at the end of a two week period during which they smoked their usual cigarette brand (visit R). Cigarettes were smoked in a laboratory free from disturbances, and the subjects were discouraged from talking while smoking. Reported daily cigarette consumption was noted at the preliminary visit; smoking record sheets were kept by the subjects for each of the control, test, and return periods.

\section{INHALED SMOKE VOLUME MEASUREMENTS}

A radiotracer technique based on that described previously by Sheahan et al $^{1617}$ was used to monitor the inhaled smoke volume, defined as the volume of smoke from the burning tip of the cigarette which was subsequently inhaled. Details of this technique have been described elsewhere. ${ }^{71819}$ Briefly, air at a flow rate of $200 \pm 10 \mathrm{ml} \mathrm{min}^{-1}$ was used to elute krypton$81 \mathrm{~m}\left({ }^{81 \mathrm{~m}} \mathrm{Kr}\right)\left(\mathrm{E}_{\gamma}=191 \mathrm{keV}, \mathrm{T}_{\frac{1}{2}}=13 \mathrm{~s}\right)$ from a generator. The krypton-air mixture was fed into a chamber of volume approximately $100 \mathrm{ml}$. A flowhead/ cigarette holder fitted into one end of the chamber; there was a one way outlet valve at the other end and

Table 1 Mean (SEM) values of smoking indices for each visit

\begin{tabular}{|c|c|c|c|c|}
\hline \multirow[b]{2}{*}{ Index (unit) } & \multicolumn{4}{|l|}{ Visit } \\
\hline & $\begin{array}{l}\text { C: usual cigarette } \\
\text { (beginning of week I) }\end{array}$ & $\begin{array}{l}\text { T1: test cigarette } \\
\text { (beginning of week 3) }\end{array}$ & $\begin{array}{l}\text { T2: test cigarette } \\
\text { (end of week 4) }\end{array}$ & $\begin{array}{l}\text { R: usual cigarette } \\
\text { (end of week 6) }\end{array}$ \\
\hline $\begin{array}{l}\text { Total inhaled smoke volume }(\mathrm{ml}) \\
\text { Total puff volume }(\mathrm{ml}) \\
\text { No of puffs } \\
\text { Mean inhaled smoke volume (ml) } \\
\text { Mean puff volume }(\mathrm{ml}) \\
\text { Puff duration }(\mathrm{s}) \\
\text { Puff interval }(\mathrm{s}) \\
\text { Total smoking time }(\mathrm{s}) \\
\text { Puff resistance }\left(\mathrm{kPa} \mathrm{s}^{-1}\right)\end{array}$ & $\begin{array}{l}323(38) \\
520(54) \\
12 \cdot 1(0 \cdot 1) \\
26 \cdot 3(2 \cdot 1) \\
43 \cdot 6(4 \cdot 1) \\
1 \cdot 9(0 \cdot 1) \\
24 \cdot 1(2 \cdot 4) \\
277(18) \\
91(4)\end{array}$ & $\begin{array}{l}309(39) \\
836(96) \\
14 \cdot 0(1 \cdot 1) \\
21 \cdot 6(1 \cdot 7) \\
58 \cdot 9(4 \cdot 1) \\
2 \cdot 1(0 \cdot 1) \\
20 \cdot 4(4 \cdot 3) \\
263(23) \\
59(1)\end{array}$ & $\begin{array}{l}312(31) \\
836(92) \\
14 \cdot 0(1 \cdot 2) \\
22 \cdot 6(1 \cdot 7) \\
59 \cdot 5(4 \cdot 7) \\
1 \cdot 9(0 \cdot 1) \\
23 \cdot 2(3 \cdot 1) \\
304(25) \\
58(1)\end{array}$ & $\begin{array}{l}264(30) \\
498(49) \\
13 \cdot 0(0 \cdot 9) \\
20 \cdot 5(1 \cdot 8) \\
37 \cdot 8(1 \cdot 9) \\
1 \cdot 5(0 \cdot 1) \\
25 \cdot 4(4 \cdot 3) \\
299(24) \\
94(5)\end{array}$ \\
\hline
\end{tabular}

$\mathrm{C}=$ control visit; $\mathrm{T} 1=$ first test visit; $\mathrm{T} 2$ = last test visit; $\mathrm{R}=$ return visit. The visits were at two week intervals.

Conversion: SI to traditional units-Puff resistance: $1 \mathrm{kPa}=7.5 \mathrm{~mm} \mathrm{Hg}$. 
a rubber sleeve enclosing the cigarette. An inner mesh prevented the sleeve from touching the cigarette but did not inhibit gas exchange within the chamber; an outer mesh surrounded the sleeve so that the chamber could be held in the hand. A puff drawn from the chamber and cigarette created a subatmospheric pressure in the chamber, which caused the closure of the outlet valve and the gradual collapse of the sleeve with negligible resistance.

Radiolabelled smoke inhaled with each puff was detected by a single probe scintillation counter placed in front of the chest and collimated to view the whole lung field. Before each cigarette was smoked a calibration was carried out to relate the maximal whole lung count rate to the volume of radiotracer inhaled. ${ }^{18}$ Only the volume drawn through the burning tip of the cigarette was radiolabelled; air drawn through ventilation holes in the cigarette filter was not and therefore did not make a contribution to the inhaled smoke volume measurement. ${ }^{19}$ Radiotracer studies were approved by the Ethical Practices Committee of the hospital and the administration of Radioactive Substances Advisory Committee. The radiation dose to subjects is less than $0.01 \mathrm{mGy}$ per cigarette and subjects gave informed consent in writing.

\section{PUFF INDEX MEASUREMENTS}

A microprocessor based system (Smoking Analyser model SAP4, Filtrona Instruments \& Automation, Ltd) made puff flow and pressure measurements as well as recordings of puff volume (integrated flow), duration, interpuff interval, and integrated pressure. Puff resistance (integrated pressure/puff volume) was subsequently calculated. This instrument includes an orifice type flowhead/cigarette holder (of negligible draw resistance) connected to two MKS223 differential pressure transducers by two meter long flexible tubes. A miniature thermocouple was incorporated in the flowhead between the cigarette filter and the orifice to sense the smoke temperature. Puff volume measurements were corrected for deviations from the flowhead calibration temperature of $22^{\circ} \mathrm{C}$ and for the $\stackrel{\widetilde{N}}{x}$ effect of density differences between pure air and $\vec{F}$ smoke laden air, which was taken to be 1.03 times as dense as pure air. ${ }^{20}$ The lighting puff was not included $\stackrel{\square}{\square}$ in any of the indices.

\section{STATISTICAL ANALYSES}

Since the sample sizes for statistical analysis were small it was not assumed that experimental data were $\vec{\circ}$ from a normal distribution; the non-parametric Wilcoxon rank sum test of pair differences was used to $\vec{\omega}$ look for significant changes between visits. ${ }^{21-23} \stackrel{2}{ }$ A probability value equal to or less than 0.05 was $\vec{x}$ taken to indicate significance. For ease of presenta- $\vec{N}$ tion, experimental data have been summarised as $u$ means and their standard deviations or standard errors.

\section{Results}

Table 1 shows the organisation of the study and the mean (SEM) of the indices for each visit. Table $2 \overrightarrow{0}$ shows the mean (SEM) of the difference in indices $\stackrel{\infty}{-}$ which were tested. In comparison with the controlo period the mean and total puff volumes increased $(p \leqslant 0.01)$ with the test cigarettes, although the mean and total inhaled smoke volumes did not changen significantly (figs 1 and 2); also there were no changesक्ष in the puff duration, puff interval, total smoking time, $\stackrel{\varrho}{\Rightarrow}$ or number of puffs. There was no significanto을 difference between the beginning and end of the test period in any index except puff duration, which decreased $(p \leqslant 0.05)$. The mean and total puff volumes on return to the usual cigarette were significantly less $(p \leqslant 0.01)$ than the values obtainedo during the test period. The indices obtained for the $e_{-}^{x}$ return period were not significantly different from 3 . those of the control period except for puff duration, which decreased $(p \leqslant 0.01)$.

The mean (SEM) daily cigarette consumptionso were similar throughout the study: control period? $21.7(1 \cdot 7)$, test period $22 \cdot 7(2 \cdot 0)$, and return period

Table 2 Mean (SEM) and significance of difference in smoking indices between visits

\begin{tabular}{|c|c|c|c|c|}
\hline \multirow[b]{2}{*}{ Index (unit) } & \multicolumn{4}{|c|}{ Difference between visits } \\
\hline & $\overline{C-T I}$ & $C-T 2$ & $T 1-T 2$ & $C-R$ \\
\hline $\begin{array}{l}\text { Total inhaled smoke volume (ml) } \\
\text { Total puff volume }(\mathrm{ml}) \\
\text { Number of puffs } \\
\text { Mean inhaled smoke volume (ml) } \\
\text { Mean puff volume (ml) } \\
\text { Puff duration (s) } \\
\text { Puff interval (s) } \\
\text { Total smoking time (s) } \\
\text { Puff resistance ( }\left(\mathrm{ka} \mathrm{s}^{-1}\right)\end{array}$ & $\begin{array}{l}14(68) \\
-316^{* * *}(170) \\
-2 \cdot 1(2 \cdot 8) \\
4 \cdot 7(5 \cdot 7) \\
-15 \cdot 2^{* * *}(8 \cdot 4) \\
-0 \cdot 2(0 \cdot 4) \\
3 \cdot 7(7 \cdot 4) \\
14(63) \\
32^{* * *}(12)\end{array}$ & $\begin{array}{l}11(103) \\
-316^{* * *}(144) \\
-1 \cdot 9(2 \cdot 6) \\
3 \cdot 7(7 \cdot 0) \\
-15 \cdot 8 * * *(6 \cdot 7) \\
0 \cdot 0(0 \cdot 2) \\
1 \cdot 0(5 \cdot 1) \\
-27(80) \\
33^{* * *}(11)\end{array}$ & $\begin{array}{l}-3(75) \\
-1(91) \\
0 \cdot 0(1 \cdot 5) \\
-1 \cdot 1(7 \cdot 0) \\
-0 \cdot 6(8 \cdot 6) \\
0 \cdot 2 *(0 \cdot 2) \\
-2 \cdot 8(8 \cdot 6) \\
-40(80) \\
1(3)\end{array}$ & $\begin{array}{l}59(91) \\
22(115) \\
-0 \cdot 9(2 \cdot 2) \\
5 \cdot 8(7 \cdot 0) \\
5 \cdot 8(10 \cdot 9) \\
0 \cdot 4^{* * *}(0 \cdot 3) \\
-1 \cdot 2(8 \cdot 1) \\
-22(80) \\
-3(11)\end{array}$ \\
\hline
\end{tabular}




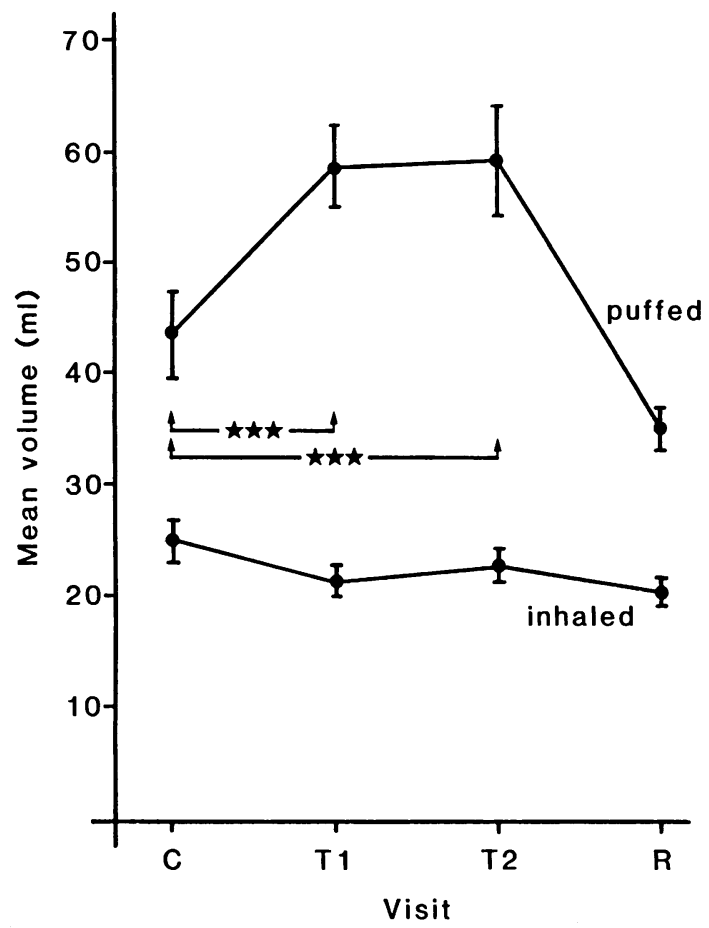

Fig 1 Mean and standard error of mean inhaled smoke and puffed volumes showing the significant increase in mean puff volume with the test cigarettes, but no change in the mean inhaled smoke volume. $C=$ control visit, $T 1=$ first test visit, $T 2=$ last test visit, and $R=$ return visit. ${ }^{* * *} p \leqslant 0.01$ with respect to visit $C$.

$21.5(1 \cdot 6)$, and did not differ significantly from that reported at the beginning of the study $(20 \cdot 8(1 \cdot 4))$.

\section{Discussion}

It is not possible to have an entirely normal environment when performing smoking experiments, and it has been reported that changes in the environmental conditions and mental state of a smoker lead to changes in the smoking manoeuvre. ${ }^{2425}$ When, however, the apparatus was used in a study of subjects smoking their usual cigarette brand on four separate occasions under the same conditions ${ }^{7}$ it was found that the smoking manoeuvre did not change from one occasion to the next. In this study the apparatus and smoking conditions were the same with all the cigarettes smoked and changes in smoking indices could be due only to the cigarettes. The absolute values of the smoking indices might have been different under different environmental conditions, though this aspect was not investigated in the present study. It is likely, however, that the differences found would be similar under other conditions.
Since the filters of the test cigarettes were ventilated it was necessary for the smokers to increase the puff volume to maintain the amount of tar or nicotine, or both, in the more dilute smoke. The finding that the mean puff volume increased when low nicotine cigarettes were smoked is consistent with results from other studies, ${ }^{4112627}$ as is the increase in total puff volume. ${ }^{428} \mathrm{~A}$ consequence of the ventilation holes is that the puff resistance is reduced, and indeed the puff resistance was lower with the test cigarette. An alternative hypothesis to the idea that a larger puff is taken because the smoke is more dilute is that the increase in puff volume is a mechanical reaction to puffing on a lower resistance. This was investigated in another (unpublished) study, in which it was found that the puff volume is determined by the smoke concentration and not by the puff resistance.

With the apparatus used in this study only the vol-

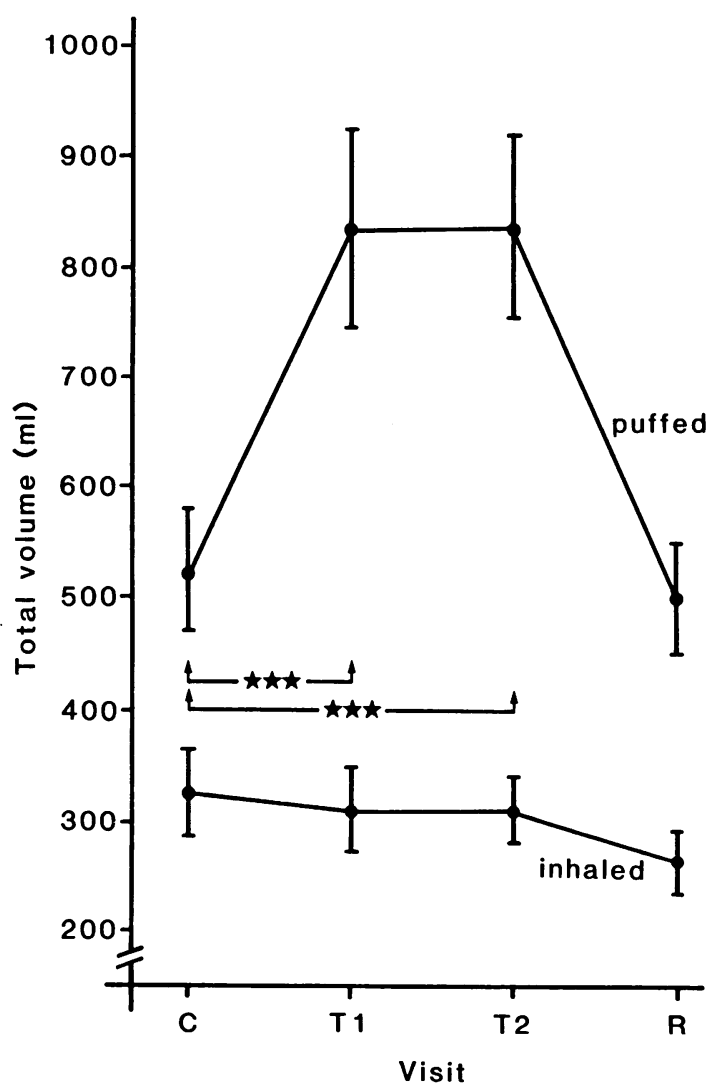

Fig 2 Mean and standard error of total inhaled smoke and puffed volumes showing the significant increase in total puff volume with the test cigarettes, but no change in the total inhaled smoke volume. $C=$ control visit, $T 1=$ first test visit, $T 2=$ last test visit, and $R=$ return visit. ${ }^{* * *} p \leqslant 0.01$ with respect to visit $C$. 
ume of smoke drawn through the tobacco filled part of the cigarette is radiolabelled. It is important to note that diluting air entering the cigarette through the ventilation holes does not affect the inhaled smoke volume indices. Neither the mean inhaled smoke volume nor the total inhaled smoke volume changed significantly with the weaker cigarette. The results of studies in which plasma nicotine concentrations were measured showed that smokers compensate for the reduction in nicotine with weaker cigarettes, ${ }^{12} 29-31$ and when ventilated cigarette holders are used. ${ }^{24}$ These results are consistent with the hypothesis that smokers compensate for the change in the concentration of tar or nicotine, or both, in the puff by altering the puff volume to regulate the amount of smoke inhaled. As observed elsewhere ${ }^{28}$ it is not possible to say what the separate effects of tar and nicotine are when both are changed; also, the tar to nicotine ratio (tar yield/nicotine yield) changed, so although the quantity of smoke did not change it is impossible to know exactly how the concentrations of the smoke constituents behaved.

Other workers have shown ${ }^{12}$ that there is no significant difference in the smoking manoeuvre from the beginning to the end of a six week period. One aspect of this study was to investigate whether the acute changes in the smoking manoeuvre seen with the first cigarette were still apparent and the same after two weeks on the test cigarette: they were. This finding suggests that it is valid to take the acute response to a new cigarette as being representative of the manner in which that cigarette will be smoked. The almost immediate response to a change in nicotine level has been found before ${ }^{27}$; it was suggested that the delivery of nicotine is controlled by the smoker from the beginning of smoking with rapid and efficient feedback mechanisms, such as the aspiration reflex. ${ }^{32}$ Although subjects switched to only weaker cigarettes in this study, it may likewise be possible to investigate the response to a cigarette higher in tar or nicotine without requiring subjects to smoke more than one test cigarette.

It might be expected that the subjects' smoking manoeuvre with their usual cigarette two weeks after finishing the test cigarette would be affected by having smoked a weaker cigarette for two weeks. This was not the case, and the smoking manoeuvre on the last visit did not differ significantly from that at the control visit, apart from a small change in puff duration. It has been shown in other studies that subjects are consistent in their smoking manoeuvre with the same type of cigarette under standard conditions ${ }^{71133}$ and return to their previous manoeuvre after changing back from a low tar, low nicotine cigarette. ${ }^{528}$

In this study the daily consumption did not change with the low tar, low nicotine cigarettes. It has been reported in other studies with this type of cigarette that the consumption over a five hour ${ }^{934}$ and four week $^{5}$ period also failed to show a statistically significant difference from that with the subjects' usual brand. In a study using ventilated cigarette듬 holders, to dilute the smoke by $20 \%$ and $60 \%,{ }^{28}$ there was no significant change in consumption between $\frac{\mathbb{}}{\alpha}$ days when the holders were and were not used. This effect was also seen in an epidemiological study with ${ }^{\text {क }}$ smokers of ventilated, unventilated, and plain ciga- $\vec{\circ}$ rettes. ${ }^{35}$

Finally, non-invasive measurements of smoke $\vec{\omega}$ inhalation and puffing with low tar, low nicotine ciga- $\overrightarrow{\overrightarrow{2}}$ rettes have shown that smokers react to the lack of tar or nicotine, or both, by taking larger puffs while $\stackrel{\vec{N}}{\mathrm{~N}}$ inhaling the same amount of smoke. This suggests that the health risk to smokers is not reduced if they $\mathrm{\sigma}^{-}$ change to a low tar, low nicotine cigarette brand. If it $\mathrm{O}$ is a lack of nicotine that is causing the smoker to take? larger puffs from the low tar, low nicotine cigarette it $\bar{z}$ may be that a low tar-medium nicotine cigarette would lead to less tar being taken into the lungs.

This study was supported by a grant from the. Tobacco Advisory Council.

\section{References}

1 Royal College of Physicians of London. Smoking or $\stackrel{\mathbb{Q}}{\mathbb{Q}}$ health. London: Pitman Medical, 1977. (Third report.) $\overrightarrow{\vec{B}}$

2 Royal College of Physicians of London. Health or $\frac{\mathrm{O}}{3}$ smoking? London: Pitman, 1983. (Follow up report.)

3 Russell MAH. Low-tar medium-nicotine cigarettes: a

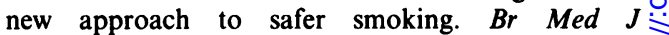
1976;i:1430-3.

4 Creighton DE, Lewis $\mathrm{PH}$. The effect of different cigarettes on smoking patterns. In: Thornton RE, ed. $\times$ Smoking behaviour, physiological and psychological 윽 influences. Edinburgh: Churchill Livingstone, 1978:289-314.

5 Rawbone RG, Murphy K, Tate ME, Kane SJ. The analysis of smoking parameters, inhalation and absorbtion 0 of tobacco smoke in studies of human smoking behaviour. In: Thornton RE, ed. Smoking behaviour, phys- $\frac{D}{2}$ iological and psychological influences. Edinburgh: Churchill Livingstone, 1978:171-94.

6 Sinclair NM. The quantitation of smoke uptake. In: Cumming G, Bonsignore G, eds. Smoking and the $\mathrm{O}$ lung. New York: Plenum Press, 1984:95-112.

7 Woodman G, Newman SP, Pavia D, Clarke SW. Inhaled o smoke volume, puffing indices and carbon monoxide uptake in asymptomatic cigarette smokers. Clin Sci 1986;71:421-7.

8 Wald N, Howard S, Smith PG, Kjeldsen K. Association between atherosclerotic disease and carboxyhaemo- $\frac{T}{\top}$ globin levels in tobacco smokers. $\mathrm{Br} \mathrm{Med} J \frac{\mathrm{P}}{\mathrm{D}}$ 1973;i:761-5.

9 Russell MAH, Wilson C, Patel UA, Feyerabend C, Cole $\stackrel{\overparen{D}}{\triangle}$ PV. Plasma nicotine levels after smoking cigarettes $\sigma$ with high, medium, and low nicotine yields. $\mathrm{Br} \mathrm{Med} J$ 1975;ii:414-6. 
10 Sutton SR, Russell MAH, Iyer R, Feyerabend C, Saloojee Y. Relationship between cigarette yields, puffing patterns, and smoke intake: evidence for tar compensation? Br Med J 1982;285:600-3.

11 McBride MJ, Guyatt AR, Kirkham ATJ, Cumming G. Assessment of smoking behaviour and ventilation with cigarettes of different nicotine yields. Clin Sci 1984;67:619-31.

12 Ashton H, Stepney R, Thompson JW. Self-titration by cigarette smokers. Br Med J 1979;ii:357-60.

13 Clarke SW, Respiratory function tests. Br J Hosp Med 1976;15:137-53.

14 Cotes JE. Lung function. 4th ed. Oxford: Blackwell Scientific, 1979.

15 Knudson RJ, Lebowitz MD, Holberg CJ, Burrows B. Changes in the maximum expiratory flow-volume curve with growth and aging. Am Rev Respir Dis 1983;127:725-34.

16 Sheahan NF, Pavia D, Bateman JRM, Agnew JE, Clarke SW. A technique for monitoring the inhalation of cigarette smoke in man, using krypton-81m. Int J Appl Radiat Isot 1980;31:438-41.

17 Sheahan NF, Pavia D, Bateman JRM, Agnew JE, Clarke SW. Objective in vivo analysis of anti-smoking cigarette filters. Thorax 1981;36:213-6.

18 Woodman G, Newman SP, Pavia D, Clarke SW. Calibration and measurement of the inhaled smoke volume in cigarette smoking. Clin Phys Physiol Meas 1985;6:251-5.

19 Woodman G, Newman SP, Pavia D, Clarke SW. An in vivo radiotracer method to allow for cigarette filter ventilation during smoking. Clin Phys Physiol Meas 1985;6:361-4.

20 Woodman G, Newman SP, Pavia D, Clarke SW. Temperature and calibration corrections to puff volume measurements in cigarette smoking. Phys Med Biol 1984;29:1437-40.

21 Siegel S. Non-parametric statistics for the behavioral sciences. Tokyo: McGraw Hill Kogakusha, 1956.

22 Armitage P. Statistical methods in medical research. Oxford: Blackwell Scientific, 1971.

23 Altman DG, Gore SM, Gardner MJ, Pocock SJ. Statistical guidelines for contributions to medical journals.
Br Med J 1983;286:1489-93.

24 Comer AK, Creighton DE. The effect of experimental conditions on smoking behaviour. In: Thornton RE, ed. Smoking behaviour, physiological and psychological influences. Edinburgh: Churchill Livingstone, 1978:76-86.

25 Ashton $\mathrm{H}$, Watson DW. Puffing frequency and nicotine intake in cigarette smokers. Br Med J 1970;iii:679-81.

26 Tobin MJ, Sackner MA. Monitoring smoking patterns of low and high tar cigarettes with inductive plethysmography. Am Rev Respir Dis 1982;126:258-64.

27 Herning RI, Jones RT, Bachman J, Mines AH. Puff volume increases when low-nicotine cigarettes are smoked. Br Med J 1981;283:187-9.

28 Sutton SR, Feyerabend C, Cole PV, Russell MAH. Adjustment of smokers to dilution of tobacco smoke by ventilated cigarette holders. Clin Pharmacol Ther 1978;24:395-405.

29 Russell MAH, Sutton SR, lyer R, Feyerabend C, Vesey CJ. Long-term switching to low-tar low-nicotine cigarettes. Br J Addict 1982;77:145-58.

30 Ebert RV, McNabb ME, McCusker KT, Snow SL. Amount of nicotine and carbon monoxide inhaled by smokers of low-tar, low-nicotine cigarettes. JAMA 1983;250:2840-2.

31 Benowitz NL, Jacob P. Daily intake of nicotine during cigarette smoking. Clin Pharmacol Ther 1984;35: 499-504.

32 Grant SGN, Woodman G, Newman SP, Pavia D, Clarke SW. Sensory mechanisms in the upper respiratory tract affect inhalation of cigarette smoke in man. Clin Sci 1986;71:117-9.

33 Adams L, Lee C, Rawbone RG, Guz A. Patterns of smoking: measurement and variability in asymptomatic smokers. Clin Sci 1983;65:383-92.

34 Russell MAH, Wilson C, Patel UA, Cole PV, Feyerabend $\mathrm{C}$. Comparison of effect on tobacco consumption and carbon monoxide absorbtion on changing to high and low nicotine cigarettes. $\mathrm{Br}$ Med $\mathrm{J}$ 1973;iv:512-6.

35 Wald NJ, Idle M, Boreham J, Bailey A. Inhaling habits among smokers of different types of cigarette. Thorax 1980;35:925-8. 